\title{
A New Adaptive Channel Reservation Scheme for Handoff Calls in Wireless Cellular Networks
}

\author{
Zhong $\mathrm{Xu}^{1}$, Zhenqiang $\mathrm{Ye}^{1}$, Srikanth V. Krishnamurthy ${ }^{2}$, Satish K. Tripathi ${ }^{2}$, \\ and Mart Molle ${ }^{2}$ \\ 1 Department of Electrical Engineering \\ 2 Department of Computer Science and Engineering, \\ University of California at Riverside, Riverside, CA92521, USA
}

\begin{abstract}
In wireless cellular networks, in order to ensure that ongoing calls are not dropped while the owner mobile stations roam among cells, handoff calls may be admitted with a higher priority as compared with new calls. Since the wireless bandwidth is scarce and therefore precious, efficient schemes which allow a high utilization of the wireless channel, while at the same time guarantee the QoS of handoff calls are needed. In this paper, we propose a new scheme that uses GPS measurements to determine when channel reservations are to be made. It works by sending channel reservation request for a possible handoff call to a neighboring cell not only based on the position and orientation of that call's mobile station, but also depends upon the relative motion of the mobile station with respect to that target cell. The scheme integrates threshold time and various features of prior schemes to minimize the effect of false reservations and to improve the channel utilization of the cellular system. Simulation results show that our scheme performs better in almost all typical scenarios than prior schemes.
\end{abstract}

Keywords: cellular networks, handoff, adaptive channel reservation

\section{Introduction}

As a mobile station (MS) 1 moves from one cell to another, its ongoing call is handed-off from the old cell to a new cell. This requires that the call be accommodated by the new cell. Since dropping a handoff call is more annoying than blocking a new call from user's perspective, handoff calls should be given higher priority than new calls. It has been shown that the method by which handoff is achieved has a significant impact on the network's performance [1]. Due to the inherent bandwidth limitation in wireless cellular networks, micro/pico cellular architectures are attractive for achieving higher system capacity [2]. In this case, the coverage area of a cell will be defined by a circular region that is a few hundred meters to a few kilometers in radius. As a direct result, the rate of handoffs increases dramatically even when MSs move at low speed.

${ }^{1}$ We use "MS" to represent "one MS with an ongoing call" in the rest of this paper.

E. Gregori et al. (Eds.): NETWORKING 2002, LNCS 2345, pp. 672684 2002.

(C) Springer-Verlag Berlin Heidelberg 2002 
The probability of an ongoing call being dropped due to a handoff failure and the probability of a new call being blocked due to the temporary unavailability of an idle channel are major metrics that define the performance of cellular systems. The handoff prioritization schemes implemented in the network have a significant impact on these two probabilities. All the handoff prioritization schemes have a common characteristic: ensuring a lower handoff dropping probability at the expense of an increased new call blocking probability. Efficient handoff prioritization schemes are those allow a high utilization of the wireless bandwidth (by accommodating a higher number of new calls) while guarantee the QoS of handoff calls.

The naive channel assignment strategy is to treat handoff calls and new calls equally [3]. This scheme would result in the new call blocking probability and the handoff call dropping probability being equal. Obviously, this scheme performs poorly when the offered load on the network is high. Much work has been done on handoff prioritization in wireless cellular systems [35/6778]. Basically there are two strategies that are popular for prioritizing handoff calls [2]: the guarded channel strategy and the handoff queueing strategy. The guarded channel strategy decreases the handoff dropping probability by reserving a fixed number of channels exclusively for handoff calls. New calls will be blocked if the number of idle channels is equal to or less than the number of guarded channels, while handoff calls can be served until all the channels are occupied. The handoff queueing strategy is a way of delaying handoff due to the temporary unavailability of channels. The mobile switching center (MSC) queues the handoff requests instead of denying access if the candidate cell has no idle channel available. Queueing is possible due to the overlapping region between adjacent cells where it can communicate with both the old and the new base station (BS). The maximum queueing time is limited by the MS' dwell time in the overlapping area. If the traffic load is heavy, or if the maximum allowed queueing time is very small, it is highly unlikely that a queued handoff request will be entertained. These two strategies can be combined to obtain better performance as compared with the individual strategies 7 .

Since the mobility behavior of different MSs may be totally different, and the traffic load offered in each cell varies from time to time, any static channel reservation scheme cannot work efficiently all the time. In order to solve this problem, several adaptive (dynamic) channel reservation schemes have been proposed [4, 5 6 7]. The shadow cluster concept proposed in [4 allows the base station of each cell to calculate the probabilities that a MS will be active in other cells at future times, and thereby facilitate the prediction of future resources demands. In [6], the number of guarded channels in each cell is adjusted according to the current estimate of the handoff call arrival rate, which is derived from the current number of ongoing calls in neighboring cells and the mobility patterns of the MSs. In [5], channels are dynamically reserved by using the request probability determined by the mobility patterns of the MSs and the current traffic load. All these schemes take into account the MSs' mobility patterns when they dynamically make channel reservations. But the mobility patterns that are considered are all 
MSs' general patterns, and they do not identify each individual MS's mobility behavior separately. In [7, the Predictive Channel Reservation (PCR) scheme is proposed and is based on mobile positioning. The threshold distance concept (See II.A for its definition) is used to define the size of channel reservation area. The PCR scheme makes predictive channel reservations for each MS based on its current position and orientation. But the threshold distance in the PCR scheme is constant for all MSs.

In this paper, we propose a new handoff prioritization scheme, which is called adaptive channel reservation (ACR) scheme. The ACR scheme integrates the features of threshold time, reservation queueing, reservation cancellation and reservation pooling to minimize the false reservations and to improve the channel utilization of the cellular system. Like [7], the ACR scheme is also based on GPS measurements [9]. We don't discuss GPS further in this paper, and just make an assumption that each MS is equipped with GPS and can obtain its position information in real-time.

The remainder of this paper is organized as follows. Section 2 outlines the ACR scheme. In section 3, we describe the models that we use for simulating the ACR scheme. Detailed performance results are presented and interpreted in section 4. Finally, we present our conclusions in section 5 .

\section{Adaptive Channel Reservation}

In the ACR scheme, channel reservation decisions are made based not only on each MS' current position and orientation, but also on the relative moving speed with respect to its next target cell. Each MS2 measures its coordinates at regular time interval (every $\Delta T$ seconds) using GPS. The coordinate information is piggybacked onto uplink data packets (or sent to the associated BS by means of special uplink packets). The BS keeps track of each MS' previous positions, predicts its trajectory [13] and calculates the relative moving speed with respect to the next cell that the MS is predicted to enter. Based on these calculations, we can predict the time within which the MS will reach this candidate cell.

In [7], the threshold distance $\left(D_{t h}\right)$ is defined as the radius of a circle which is co-centered with a cell, and this circle is smaller than the cell's coverage area (Figure1(a)). The area between these two circles is called the channel reservation area. When a MS enters the reservation area of a cell from the inner part of that cell (or a new call is generated inside the reservation area), and at the same time, is heading to a new cell, a reservation request will be sent to that new cell's BS. There are some problems in the PCR scheme. Suppose a MS moves into the reservation area of cell A and heads to cell C (See Figure 1(a)); although the $\mathrm{MS}$ is located in the reservation area of cell $\mathrm{A}$, there is a long distance between the MS's current location and the boundary of cell C. After the MS reserves a channel, it may need a long time to move into cell $\mathrm{C}$. In this case, the time for which a channel is reserved for this MS will be too long, and thus the overall channel utilization will deteriorate. Another problem is that each MS has its own

\footnotetext{
${ }^{2}$ We assume that every MS can carry at most one call at a time in this paper.
} 


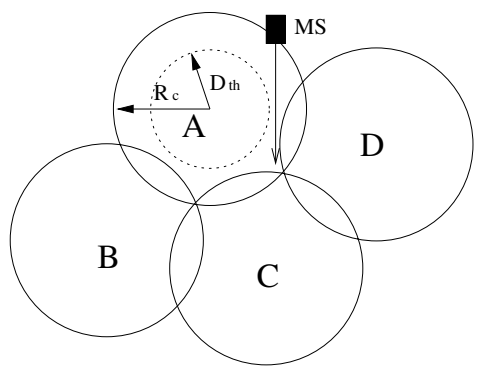

(a)

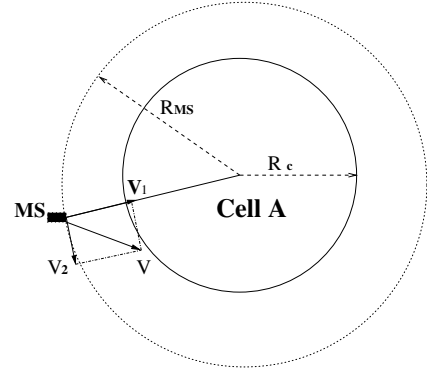

(b)

Fig. 1. Threshold distance in the PCR scheme (a) and threshold time in the ACR scheme (b)

motion pattern and hence it is inappropriate to define one constant threshold distance for all MSs. One extreme example is that there is a MS located in the overlapping area of two adjacent cells, the moving speed of this MS is very slow (close to 0). If the PCR scheme is used, two channels (each cell has one channel occupied) will be occupied by this call, one channel is used for communication in the current cell and the other is reserved for this call in the adjacent cell. Since the MS of this call is almost stationary, the reserved channel may not be used for the life time of this call. Naturally, this method leads to the under-utilization of wireless channels.

\subsection{Threshold Time}

In order to solve these problems, we use threshold time $\left(T_{t h}\right)$ instead of threshold distance to reflect possible reservation requests. Here $T_{t h}$ is a constant time value. According to each MS' current moving speed, orientation and location information, BSs can predict the time within which the MS will reach the boundary of its next target cell. In Figure 1(b), a MS is moving with a velocity $V$ towards cell A. The velocity $V$ can be decomposed into two orthogonal component vectors, $V_{1}$ and $V_{2}$, where $V_{1}$ is the velocity component of this MS towards the center of cell $A$. From $V_{1}$ and $R_{M S}$ (the distance between the MS and the center of cell $A$ ), we can estimate the time $T_{p}$ by which the MS will reach the boundary of cell A.

$$
T_{p}=\frac{R_{M S}-R_{c}}{V_{1}} .
$$

where $R_{c}$ is the radius of cell $\mathrm{A}$.

If $T_{p}>T_{t h}$, it means that the MS may take a time longer than $T_{t h}$ to reach cell $\mathrm{A}$, and it does not need a channel reservation in that cell at current time. If $T_{p} \leq T_{t h}$, it means that the MS under consideration will move into cell $A$ soon, and a reservation request will be sent by the current BS to cell $A$ 's BS. Suppose $R_{t h}=\left.R_{M S}\right|_{T_{p}=T_{t h}}$; we call $R_{t h}$ the threshold distance for this MS. Note that 
the threshold distance defined in our paper is different from that in 7 in that different MSs have different threshold distances even though all the MSs have the same $T_{t h}$, because they have different relative moving speeds.

Like the PCR scheme, in our scheme, threshold time is integrated with reservation queueing, reservation cancellation and reservation pooling to minimize the effect of false reservations and to improve the channel utilization of cellular systems. In the following paragraphs, we briefly describe the concepts of reservation cancellation, reservation pooling and reservation requests queueing defined in 7].

\section{$2.2 \quad$ Reservation Requests Queueing}

If a reservation request is received by the $\mathrm{BS}$ of one cell, and there is no idle channel available, this reservation request will be put into a reservation queue. If the reservation queue is not empty, a channel released by a call (either complete or handed-off to a neighboring cell) is added to the reservation pool at once and one reservation request is dequeued.

\subsection{Reservation Cancellation}

A reservation may be invalid (false reservation) at a later time because the MS may change its moving direction, slow down its moving speed or because the call may terminate before the MS reaches the candidate cell. In this case, the false reservation will be canceled and a reserved channel will be released (if the reservation queue is empty) or one reservation request is deleted from the reservation queue (if the reservation queue is not empty). The frequency of occurrence of false reservations depends primarily on the MSs' mobility pattern and prediction accuracy of future movement.

\subsection{Reservation Pooling}

Rather than strictly mapping each reserved channel to the MS that made the reservation, the set of reserved channels, at any moment, is used as a generic pool to serve handoff requests. Once a handoff is needed, the BS will randomly choose a reserved channel from the reservation pool and assign it to the handoff call. So when one BS sends a reservation request to another BS, it does not need to send the MS' ID.

The overhead incurred by the ACR scheme is the prediction of each MS' future trajectory, the transmission of reservation requests and reservation cancellation messages among BSs. Because all of these functions can be performed by BSs, there is no extra overhead for MSs (for which computation power is limited). The communication overhead (among the BSs) is transmitted over wire-line network, and does not consume the precious wireless bandwidth. 


\section{Simulation Model}

We construct a simulation model to evaluate the performance of the ACR scheme. The model is implemented in CSIM18 [14]. This simulation model includes system topology model (cell model), traffic model and user mobility model.

\subsection{Cell Model}

The simulation is conducted over a $L$ layer micro cellular mobile radio system (See Figure 21). Square, circular or hexagonal cells are commonly used in the simulation of wireless cellular systems. In our simulation we use hexagons to represent the neighborhood relationships among cells and circles to approximate the coverage area of cells. There are overlapping areas between adjacent cells. The radius of each circle (or hexagon) is represented by $R_{c}$. There is one central cell in the topology (first layer). The central cell is surrounded by six cells which make up the second layer. There are 12 cells in the third layer, and $6(i-1)$ cells in the $i$ th layer $(1<i \leq L)$.

In order to avoid border effects, when a MS moves out of the service area

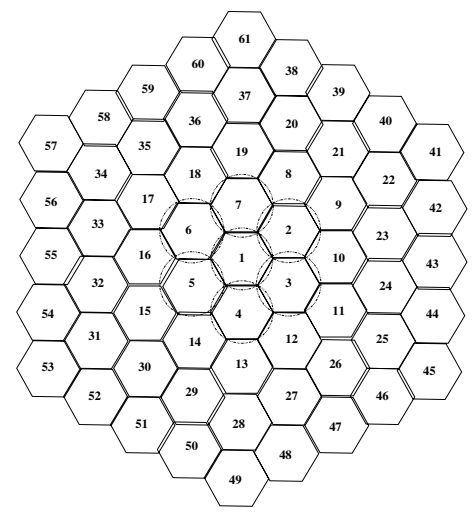

Fig. 2. The topology of a 5-layer wireless cellular system

of the system, this MS will be wrapped around to re-enter the system from the other side. Such a toroidal arrangement is an efficient way to approximately simulate very large cellular systems.

\subsection{Traffic Model}

In our simulation model we only consider homogeneous calls, and assume that each MS needs only one channel per call. Call generation in the system follows a 
Poisson process with an average arrival rate $\lambda$. The call holding time $T_{c}$ follows an exponential distribution with an average service rate $\mu_{c}$. The number of channels in each cell is a constant $c$. The normalized offered traffic load of the system is defined to be

$$
\frac{\lambda}{\mu_{c} \cdot N \cdot c},
$$

where $N$ is the number of cells in the system, and is given by:

$$
N=1+\sum_{i=2}^{L} 6(i-1)=3 L(L-1)+1 .
$$

Note that the load is measured in Erlang.

\subsection{Mobility Model}

Several mobility models, such as the random-walk model and the fluid-flow model are often used to depict MS' moving behavior in simulations and analyses 1011 , 12. In our simulation, we consider a more realistic mobility model. When a new call is generated, the MS initially chooses a speed which is uniformly distributed over $\left[V_{\min }, V_{\max }\right]$ and a moving direction which is uniformly distributed over $\left[0,360^{\circ}\right.$ ). In each variable-length period $T_{u}$ (which is exponentially distributed with mean $E\left[T_{c}\right] / 3$ ), the MS moves along a straight line. After that period, the MS may stop (with a probability $P_{\text {stop }}$ ) for a time $T_{u}$ or continue to move (with a probability $\left.P_{\text {cont. }}=1-P_{\text {stop }}\right)$. If the MS continues to move, it may change its moving direction. The MS makes $\pm 90^{\circ}$ turns with probability $P_{90^{\circ}}$, makes $\pm 45^{\circ}$ turns with probability $P_{45^{\circ}}$, and moves along the original moving direction with probability $P_{0^{\circ}}=1-\left(P_{90^{\circ}}+P_{45^{\circ}}\right)$.

In order to evaluate the effects of speed patterns on the system performance, three different speed patterns are defined.

- V1: $V_{\min }=0$ and $V_{\max }=20 \mathrm{~m} / \mathrm{s}$.

- V2: $V_{\min }=0$ and $V_{\max }=5 \mathrm{~m} / \mathrm{s}$.

- V3: $V_{\min }=15 \mathrm{~m} / \mathrm{s}$ and $V_{\max }=20 \mathrm{~m} / \mathrm{s}$.

Compared to V1, V2 is low speed moving pattern, and V3 is high speed moving pattern.

\section{Performance Evaluation}

We define the new call blocking probability $P_{b}$, the handoff dropping probability $P_{d}$ and the call incompletion probability $P_{n c}$ as the system performance metrics. Call incompletion probability is the probability that a call is not completed (either due to being blocked or because of being dropped during handoff). The values of the various parameters used in simulation are listed in Table 1 . In order to evaluate the performance of the ACR scheme, we simulate the PCR scheme with the same simulation model and under the same system conditions, and compare its performance results with that of the ACR scheme. 
Table 1. Parameter Values in the Simulation

\begin{tabular}{c|c|c}
\hline Parameter & Value & Description \\
\hline \hline$L$ & 5 & Cell Layer Number \\
\hline$R_{c}$ & $500 \mathrm{~m}$ & Cell Radius \\
\hline$c$ & 20 & Number of Channels in each Cell \\
\hline$T_{c}$ & $180 \mathrm{~s}$ & Call Holding Time \\
\hline$P_{\text {stop }}$ & 0.1 & Probability with which a MS stops \\
\hline$P_{\text {cont. }}$ & 0.9 & Probability with which a MS continues motion \\
\hline$P_{0^{\circ}}$ & 0.7 & Probability of Keeping Original Moving Direction \\
\hline$P_{45^{\circ}}$ & 0.1 & Probability of Making a $45^{\circ}$ turn \\
\hline$P_{90^{\circ}}$ & 0.2 & Probability of Making a $90^{\circ}$ turn \\
\hline
\end{tabular}

Figure 3 shows the $P_{b}, P_{d}$ and $P_{n c}$ experienced by the system when the ACR scheme is used with different values of $T_{t h}$. The MSs move in accordance to the speed pattern V1. From Figure 3 (a) it is seen that $P_{d}$ decreases from $0.17 \%$ to $0.025 \%$ with the increase of $T_{t h}$ (from 3 seconds to 20 seconds) when normalized traffic load is 0.9 ; the penalty incurred is that, $P_{b}$ increases from $20 \%$ to $29 \%$ (See Figure 3(b)). We also find that $P_{d}$ is already very small (compared to $P_{b}$ ) even when $T_{t h}$ is very small (for example, $T_{t h}=3$ seconds). The reason for this result is that the overlapping area of a cell with its neighboring cells contributes a fairly large portion (about 35\%) of the entire cell, and even if a MS can not access an idle channel before it traverses the boundary of its next cell, it still has a certain period of time (its dwell time in the overlapping area) to wait for an idle channel. So its maximum allowed channel waiting time is larger than $T_{t h}$. Since $P_{d}$ is much smaller than $P_{b}$, most of the unsuccessful calls are new calls (which are blocked), therefore the call incompletion probability $P_{n c}$ and the new call blocking $P_{b}$ are almost the same (See Figure 3(c)).

Figures 4 and 5 show the performance of the ACR scheme under two different speed patterns (low speed pattern V2 and high speed pattern V3). From these two figures, it is seen that $P_{d}$ under the low speed pattern is a little higher than that under the high speed pattern. On the other hand, $P_{b}$ is lower under the low speed pattern. Under the low speed pattern, the possibility that an ongoing call is handed-off to another cell is smaller than that under the high speed pattern. As a result, under the low speed pattern, the number of handoffs is smaller, and consequently, the number of reserved channels at any given time is also smaller. Since the ACR scheme is incorporated with reservation pooling, the more the number of reserved channels, the lower the probability $P_{d}$. This is the reason for $P_{d}$ being high and $P_{b}$ being low under the low speed pattern. Another observation is that both $P_{d}$ and $P_{b}$ are not very sensitive to a change in $T_{t h}$ under the low speed pattern. On the other hand, the sensitivity is much higher under the high speed pattern.

The comparison of the ACR scheme with the PCR scheme in terms of performance under the speed pattern V1 is shown in Figure 6. By choosing $T_{t h}=3$ seconds in the ACR scheme and the threshold distance $D_{t h}=0.723 R_{c}$ in the PCR scheme, the two schemes have almost the same $P_{d}$ for various normalized traffic loads (See Figure 6(a)). The ACR scheme decreases $P_{b}$ by $4.5 \%$ as compared with the PCR scheme (See Figure [6(b)). In other words, for a given 


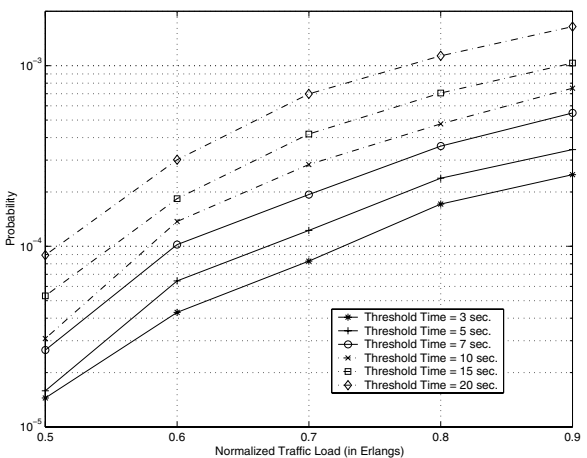

(a) $P_{d}$

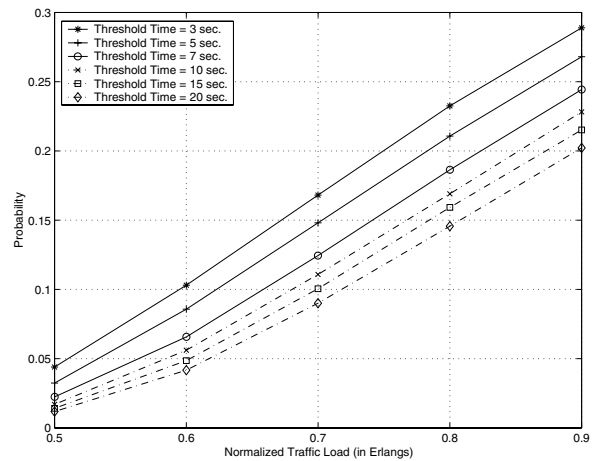

(b) $P_{b}$

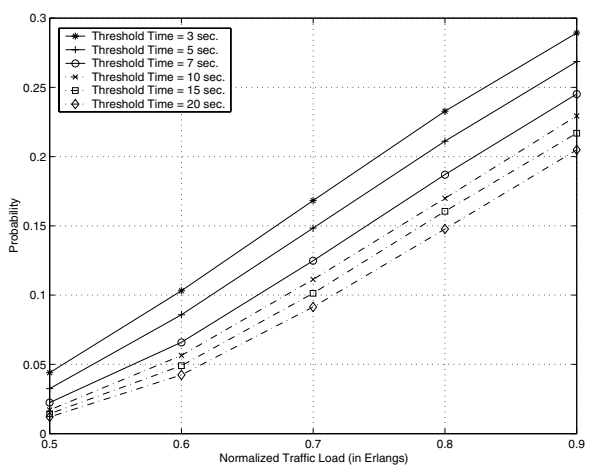

(c) $P_{n c}$

Fig. 3. Performance of the ACR scheme under speed pattern V1

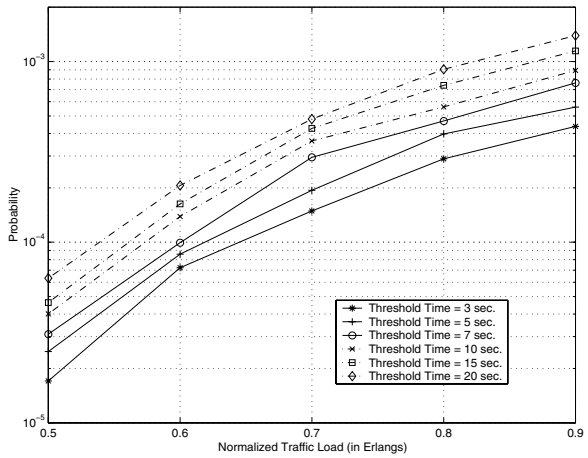

(a) $P_{d}$

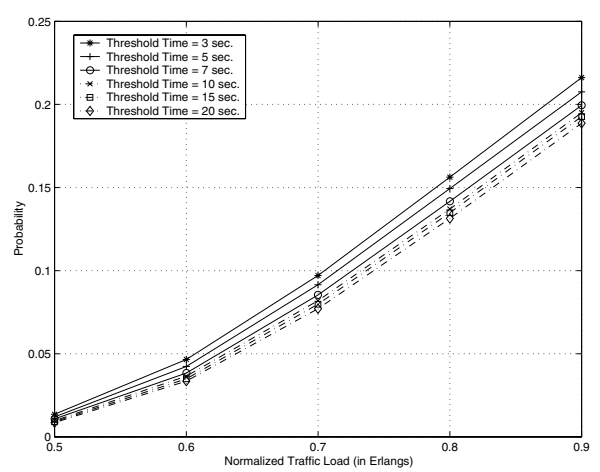

(b) $P_{b}$

Fig. 4. Performance of the ACR scheme under speed pattern V2 (low speed pattern) 


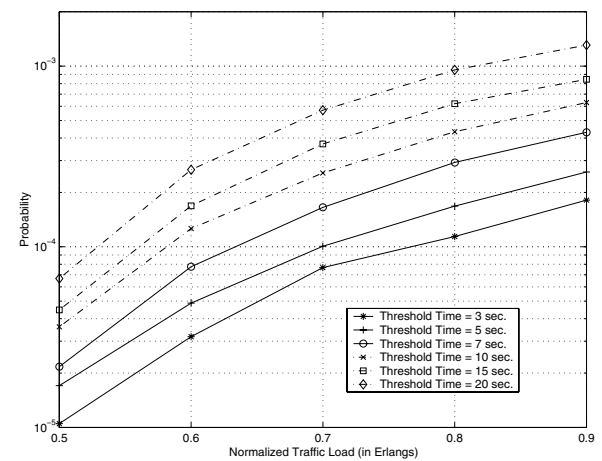

(a) $P_{d}$

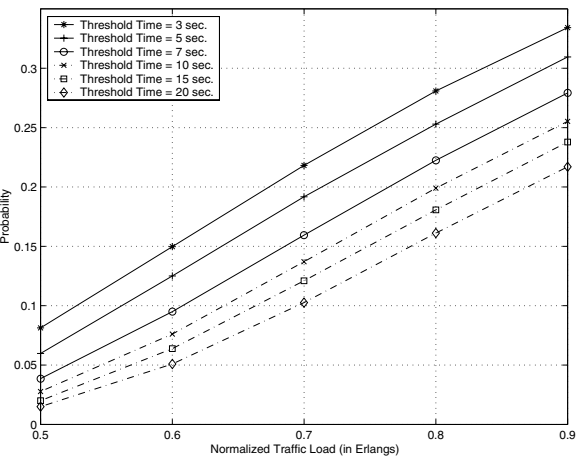

(b) $P_{b}$

Fig. 5. Performance of the ACR scheme under speed pattern V3 (high speed pattern)

normalized traffic load, the ACR scheme allows a higher number of calls (approximately $4.5 \%$ more ) than the PCR scheme, while maintains the same handoff call dropping probability $P_{d}$. Similarly, by choosing $T_{t h}=20$ seconds in the ACR scheme and $D_{t h}=0.69 R_{c}$ in the PCR scheme, the two schemes have the same $P_{d}$. Correspondingly the value of $P_{b}$ in the ACR scheme is lower by $1.5 \%$ as compared with that seen in the PCR scheme. Since the call incompletion probability $P_{n c}$ is dominated by $P_{b}$, the ACR scheme can ensure more completed calls than the PCR scheme. Consequently, the ACR scheme achieves a higher channel utilization than the PCR scheme.

Figure 7 compares the performance of the two schemes under the low speed pattern V2. In Figure 7(a), by choosing $T_{t h}=3$ seconds and $D_{t h}=0.815 R_{c}$, these two schemes have almost the same $P_{d}$ for various normalized traffic loads. Correspondingly, in Figure 7 (b), $P_{b}$ in the ACR scheme is lower by $1 \%$ as compared with that in the PCR scheme. Similarly, by choosing $T_{t h}=20$ seconds and $D_{t h}=0.76 R_{c}$, the two schemes have almost the same $P_{d}$, and $P_{b}$ in the ACR scheme is lower by $1.5 \%$ as compared with that in the PCR scheme. One interesting observation is that $P_{b}$ in the ACR scheme is much lower with a larger $T_{t h}$ under speed pattern V2 (In contrast, $P_{b}$ is somewhat higher if $T_{t h}$ is larger as seen in Figure 6(a)). Under the low speed pattern, the average number of channel reservation requests is smaller than that under the high speed pattern, while the channel holding time of a call in a given cell is longer. This would imply that the rate at which the occupied channels are released will be smaller if we have a low speed pattern and the reservation request may therefore need a longer time to get an idle channel. For the same value of $T_{t h}$ and the same normalized traffic load, $P_{d}$ under a low speed pattern is higher and $P_{b}$ is lower than the corresponding values observed under a high speed pattern.

Figure 8 compares the performance of these two schemes under the high speed pattern. In Figure 8(a), by choosing $T_{t h}=3$ seconds and $D_{t h}=0.71 R_{c}$ we ensures that the two schemes have almost the same $P_{d}$ for various normalized traffic loads. In Figure 8 b), we see that $P_{b}$ in the ACR scheme is lower by $4 \%$ 
as compared with that seen in the PCR scheme. On the other hand, the ACR scheme has almost the same $P_{d}$ and $P_{b}$ as the PCR scheme when $T_{t h}=15 \mathrm{sec}-$ onds and $D_{t h}=0.6 R_{c}$. This is due to the fact that under high speed patterns, if $T_{t h}$ is large, the channel reservation area will become very large; consequently the fraction of calls that make reservation requests in adjacent cells will be large. The performance of the ACR scheme will therefore deteriorate. However, even in this unrealistic scenario, the ACR scheme still performs as well as the PCR scheme.

Since the ACR scheme is distributed, it can be applied not only in homogeneous

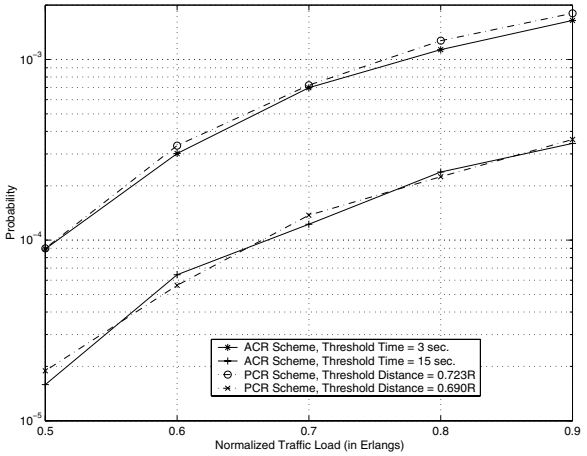

(a) $P_{d}$

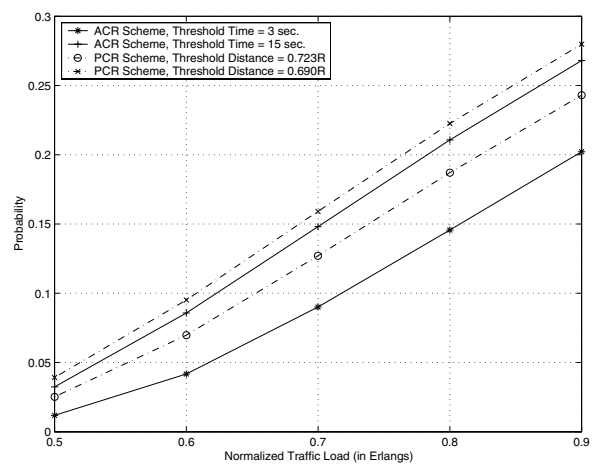

(b) $P_{b}$

Fig. 6. Performance comparison between the ACR scheme and the PCR scheme under V1

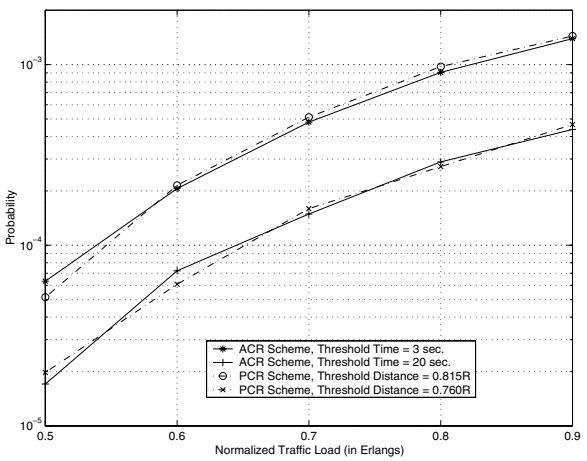

(a) $P_{d}$

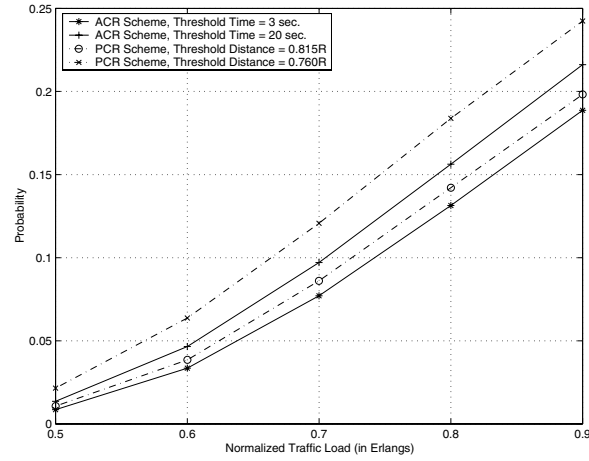

(b) $P_{b}$

Fig. 7. Performance comparison between the ACR scheme and the PCR scheme under V2 


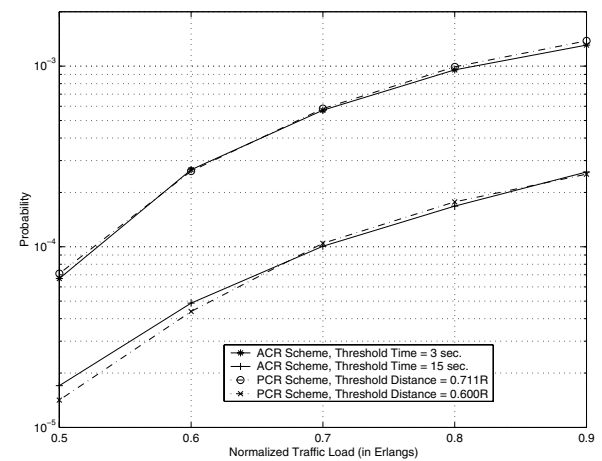

(a) $P_{d}$

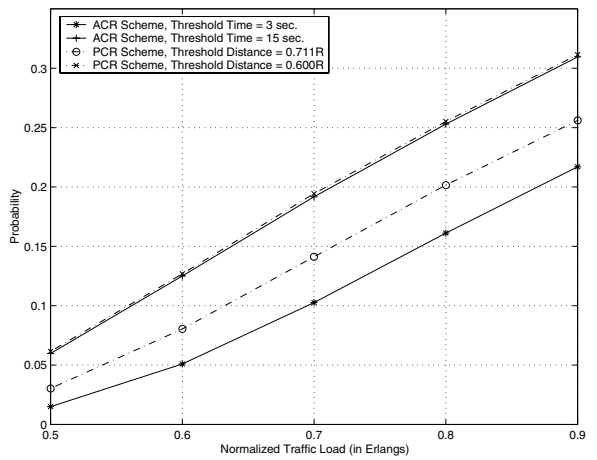

(b) $P_{b}$

Fig. 8. Performance comparison between the ACR scheme and the PCR scheme under $\mathrm{V} 3$

systems in which every cell has the same size, shape and number of channels, but also in heterogeneous systems in which each cell might have a different coverage area, a different shape and different number of channels. The scheme may be expected to work well under non-uniform traffic loads as well.

\section{Conclusion}

In this paper, we propose an adaptive channel reservation (ACR) scheme for handoff prioritization which is based on GPS measurement. In this scheme, a base station sends a reservation request to a neighboring cell not only in accordance to the position and orientation of a mobile station, but also by taking into account the mobile station's relative moving speed with respect to its next target cell. The scheme introduces a new concept called the threshold time, and uses this in conjunction with other prior concepts such as reservation queueing, reservation cancellation and reservation pooling to minimize the effect of false reservations and to improve the channel utilization of the cellular systems. Extensive simulations were performed, and the simulation results show that, the ACR scheme can accommodate more new calls (has lower new call blocking probability $P_{b}$ ) than the PCR scheme while maintaining the same value of handoff call dropping probability $P_{d}$ for any given traffic load.

\section{References}

1. S. Tekinay and B. Jabbari; "Handover policies and channel assignment strategies in mobile cellular networks," IEEE Communications Magazine Vol.29, No.11, 1991.

2. N.D. Tripathi, J.H. Reed and H.F. VanLandinoham; "Handoff in cellular systems" IEEE Personal Communications Vol.5, No.6, Dec. 1998.

3. D. Hong and S.S. Rappaport; "Traffic model and performance analysis for cellular mobile radio telephone systems with prioritized and non-prioritized handoff procedures" IEEE Trans. on Veh. Tech., Aug. 1986 
4. D. A. Levine, I. F. Akyildiz and M. Naghshineh; "A Resource Estimation and Call Admission Algorithm for Wireless Multimedia Networks Using the Shadow Cluster Concept." IEEE/ACM Transactions on Networking, Vol. 5, No.1, February 1997

5. Y.C. Kim, D.E. Lee; "Dynamic Channel Reservation Based on Mobility in Wireless ATM Networks" IEEE Communications Magazine, Nov. 1999.

6. O. T. Yu and V. C. M. Leung; "Adaptive Resource Allocation for Prioritized Call Admission over an ATM-Based Wireless PCN" IEEE Journal on Selected Areas in Communications, Vol.15, No.7, September 1997

7. M.H. Chiu and M.A. Bassiouni; "Predictive Schemes for Handoff Prioritization in Cellular Networks Based on Mobile Positioning" IEEE Journal on Selected Areas in Communications, Vol.18, No.3, March 2000

8. W. Zhuang, K.C. Chua and S.M. Jiang; "Measurement-Based Dynamic bandwidth Reservation Sheme for Handoff in Mobile Multimedia Networks" IEEE 1998 International Conference on Universal Personal Communications. Conference Proceedings

9. B. Hofmann-Wallehnhof, H. Lichtenegger and J. Collins; "Global Positioning System: Theory and Practice", Springer-Verlag, 1997.

10. B. Liang and Z.J. Hass; "Predictive Distance-Based Mobility Management for PCS Networks" IEEE INFOCOM'g9

11. M. M. Zonoozi and P. Dassanayake; "User Mobility Modeling and Characterization of Mobility Patterns" IEEE Journal on Selected Areas in Communications, Vol. 15, No. 7, Sept. 1997.

12. H. Xie, S. Tabbane, and D.J. Goodman; "Dynamic Location Area Management and Performance Analysis" Proceedings of 43rd IEEE Vehicular Technology Conference May 1993.

13. W. Cui. and X. Shen; "User Movement Tendency Prediction and Call Admission Control for Cellular Networks" 2000 IEEE International Conference on Communications. ICC 2000

14. http://www.mesquite.com/ "CSIM18 Introduction" 